OPEN ACCESS

Edited by:

Francesca Marina Bosco,

University of Turin, Italy

Reviewed by:

Anna Mulasso,

University of Turin, Italy

Pamela Wicker,

German Sport University Cologne,

Germany

*Correspondence:

Nadja Schott

nadja.schott@inspo.uni-stuttgart.de

Specialty section:

This article was submitted to

Cognitive Science,

a section of the journal

Frontiers in Psychology

Received: 17 December 2018

Accepted: 23 May 2019

Published: 07 June 2019

Citation:

Schott N and Krull K (2019) Stability of Lifestyle Behavior The Answer to Successful Cognitive Aging? A Comparison of Nuns, Monks, Master Athletes and Non-active Older Adults.

Front. Psychol. 10:1347.

doi: 10.3389/fpsyg.2019.01347

\section{Stability of Lifestyle Behavior - The Answer to Successful Cognitive Aging? A Comparison of Nuns, Monks, Master Athletes and Non-active Older Adults}

\author{
Nadja Schott* and Katja Krull \\ Department of Sport and Exercise Science, University of Stuttgart, Stuttgart, Germany
}

Background: Epidemiological studies of the effect of physical activity on cognition demonstrated an inverse relationship between physical activity and cognitive decline. However, such health behaviors are hardly invariable over time. The relative homogeneity of the adult lifestyle of nuns/monks as well as master athletes reduces the likelihood of confounding due to differences in their participation in regular life-long physical activities. The purpose of this study was to determine if there were differences in cognitive functions between nuns/monks, master athletes and sedentary, but otherwise healthy older adults. Additionally, we examined associations between demographic variables (education, sex, age), BMI, physical activity, exercise, and fitness and cognitive performance.

Methods: We recruited three groups of healthy participants without cognitive deficits: (1) Nuns/Monks ( $n=20$; age $77.5 \pm 5.56 ; 5 \mathrm{M}, 15 \mathrm{~W}$ ), (2) Master Athletes $(n=20$; age $76.5 \pm 5.33 ; 12 \mathrm{M}, 8 \mathrm{~W})$, and (3) Sedentary $(n=20 ; 76.4 \pm 5.96,6 \mathrm{M}, 14 \mathrm{~W})$. Cognitive performance (working memory, inhibition) was measured with a n-back task and a flanker task, participation in physical activities with the "German-PAQ-50+," and physical fitness with the 30s chair stand and arm curl test.

Results: As predicted, ANOVA comparing groups revealed the three groups differed in cognition, physical activity, and physical fitness with inactive older adults performing lower on all tests than the other two groups. Hierarchical regression analyses showed a positive influence of lifestyle stability on accuracy and reaction time for working memory and inhibitory performance. The highest correlation coefficients for fitness and cognitive performance emerged for the group of nuns and monks.

Conclusion: Life-long stability of an active lifestyle may confer benefits to some aspects of working memory, attention, and inhibitory control. Longitudinal studies are recommended to further examine the causal relationship of lifestyle stability and cognitive function in such specific cohorts.

Keywords: master athletes, working memory, attention and inhibitory control, lifestyle engagement, older adults 


\section{INTRODUCTION}

Worldwide we find an increasing number of individuals becoming centenarians and live "sharp as a tack" and/or physically fit while many others suffer varying degrees of cognitive and motor deterioration with increased morbidity, dependence, and mortality (Negash et al., 2013). Having a low risk of disease and disease-related disability, high cognitive, and physical functional capacity, and being actively engaged with life have been agreed upon as essential components of successful aging (Rowe and Kahn, 1997). Studies have also shown that healthy lifestyles are even as influential as genetic factors in helping older adults to attenuate the age-related limitations (Rea, 2017). Common behaviors contributing to healthy aging are eating in moderation, sufficient hydration, exercise, purposeful living (e.g., life philosophy, volunteerism, "hard work"), spirituality, and maintaining social support systems (Pignolo, 2018).

Physical activity, exercise and fitness ${ }^{1}$ are key factors in maintaining or even improving cognitive performance. A number of studies have found that high physical activity levels or better low levels of sedentariness (prolonged and uninterrupted periods of sitting) as well as high aerobic capacity are associated with neuroprotective effects on structural and functional brain health (e.g., increased gray and white matter of the prefrontal and temporal cortices, neurogenesis, synaptogenesis) in healthy older adults as well as patients with Mild Cognitive Disorder or Dementia (for a recent review see Tyndall et al., 2018). Despite numerous studies in humans and animals, the mechanisms for the effect of exercise on cognitive control are unclear. However, some aspects of the process, such as increased astrocyte glycogen storage, increased expression of BDNF, PGC- $1 \alpha$ signaling and altered IL- 6 production of the skeletal muscle, seem to mediate the benefits of exercise for cognitive function (Norman et al., 2018).

Studies examining the relationship between physical activity, exercise, fitness and cognition usually included individuals who were not particularly inclined to physical activity and exercise or were shown to rather exercise irregularly. Despite the knowledge of healthy lifestyle components only $\sim 36 \%$ of older adults in the United States (Keadle et al., 2016) and $~ 58 \%$ in Europe (Marques et al., 2015) meet the physical activity guidelines of $150 \mathrm{~min} /$ week of moderate physical activity and/or $75 \mathrm{~min} /$ week of physical activity. Master athletes (MA; exercise on a regular basis to compete in organized competitive sport) on the other hand, maintain high levels of systematic exercise and retain better physical function, muscular strength and body fat levels than age-matched non-athletes (McKendry et al., 2018). They have been proposed as an excellent model for the best attainable trajectory of aging (Geard et al., 2017; Lazarus and Harridge, 2017). Only few studies have examined brain function and

\footnotetext{
${ }^{1}$ Physical activity is defined as any bodily movement produced by muscle action that increases energy expenditure. Physical exercise implies planned, structured, repetitive and purposeful physical activity, often with the goal of improving or maintaining one's physical fitness. Physical fitness is a physiological state that provides the ability to carry out activities of daily living with optimal performance, endurance, and strength (Caspersen et al., 1985).
}

cognitive performance in MA compared to the typically more or less active older adult (Taran et al., 2013; Thomas et al., 2013; Tseng et al., 2013; Alfini et al., 2016; Zhao et al., 2016; Wouters et al., 2017; Dupuy et al., 2018). MA demonstrated greater resting cerebral blood flow in the posterior cingulate cortex (Thomas et al., 2013), higher gray and white matter concentrations in the right parietal and occipital lobes (Tseng et al., 2013); better executive control in a dual-task (Dupuy et al., 2018), but no relationship between physical activity and working memory, executive function, and visuospatial short-term memory (Wouters et al., 2017).

In addition to physical activity, other healthy behaviors such as religious (beliefs and practices related to the Transcendent) and/or spiritual (intrinsic part of being human; comprises a sense of connectedness to others) involvement as well as mindfulness meditation (awareness that arises through paying attention, on purpose, in the present moment, nonjudgmentally) influences various body-mind mechanisms (Koenig, 2012; Baer, 2019). A growing body of research shows that these forms of healthy behaviors reduce stress, depression, pain and anxiety, improves attention and memory, and promotes self-regulation as well as empathy (Malinowski et al., 2015; Geiger et al., 2016; Gonçalves et al., 2017). That includes evidence suggesting that different forms of religious and spiritual behavior as well as mindfulness meditation may protect the brain from normal cortical thinning (a sign of cognitive aging) and improve cognitive performance in elderly people (Giordano and Engebretson, 2006; Miller et al., 2014; Muehsam et al., 2017). Two iconic studies that have been key to advancing our knowledge of changes in motor and cognitive performance are the Religious Order Study and the Nun Study (Snowdon, 2003; Wilson et al., 2012; Latimer et al., 2017; Bennett et al., 2018). The Religious Order Study comprises $n=1240$ Catholic nuns, priests, and brothers (Wilson et al., 2012), while the Nun Study comprises $n=678$ Roman Catholic School Sisters of Notre Dame (Latimer et al., 2017). Using a time-varying effects model, Boyle et al. (2017) showed for data from the Religious Orders Study and the Rush Memory and Aging Project that a global motor measure declined with a mean 0.024-unit per year until a mean of 2.46 years before death when rate of decline increased nearly fivefold to -0.117 -unit per year. Furthermore, the authors described that a global cognitive measure declined with a mean of 0.027 -unit per year until a mean of 2.76 years before death when rate of decline increased more than 13-fold to -0.371-unit per year (Boyle et al., 2017). In comparison with the Nun Study, healthy older adults from the Honolulu-Asia Aging Study were found to have differences in prevalence of Alzheimer disease neuropathologic change, small vessel vascular brain injury, and Lewy body disease (Latimer et al., 2017). The authors suggest that lifestyle factors are responsible for their results in addition to gender and ethnicity.

Such health lifestyle behaviors are hardly invariable over time, adherence to a constant healthier lifestyle is moderate at best (Petersen et al., 2015). The relative homogeneity of the adult lifestyle of nuns/monks as well as master athletes reduces the likelihood of confounding due to differences in their participation in regular life-long physical activities. For example, due to their specific living conditions, members of religious orders are much 
more homogeneous than any other population group with regard to many health-relevant aspects. Nuns and monks maintain a lifestyle determined by vows with an almost identically regulated daily routine regarding sleep rhythm, working hours, physical and mental activities as well as phases of rest. In addition, all members of the order have almost identical living conditions, a comparable diet and the same access to medical care. In comparison to the typical population, nuns and monks cultivate a life largely free of social stress factors. They do not have to provide for themselves or a family and do not suffer from marital problems and financial burdens or worries in connection with child-raising and individual old-age provision. Results from the German-Austrian Cloister-Study show that $33.1 \%$ of the nuns and monks surveyed are active in sports more than once a week; a further $36.8 \%$ state that they carry out relaxation and meditation exercises every day; $54.7 \%$ describe their health status as excellent, and $20.8 \%$ often feel nervous or stressed (Wiedemann et al., 2014).

Several hypotheses and models have been advanced to account for a healthy lifestyle-induced cognitive protection (e.g., through exercise, well-balanced diet, minimized psychosocial stress): "brain reserve capacity" (Satz, 1993), "cognitive reserve capacity" (Stern, 2002), "neurocognitive scaffolding” (ReuterLorenz and Park, 2014), and recently the "Adaptive Capacity Model” (Raichlen and Alexander, 2017). The brain reserve capacity is defined as the brain's resilience to pathological damage, for example, a lesion might lead to impairment in one individual with a low brain reserve capacity, but not in another individual with a higher brain reserve capacity (Christie et al., 2017). Cognitive reserve capacity refers to the attempt of the brain to compensate and to adapt following the onset of brain pathology (for a recent discussion see Nilson and Lövdén, 2018). However, both concepts are described as still hypothetical due to the only indirect measures of its operation (see for an extended discussion Dixon and Lachman, 2019). The Scaffolding Theory of Aging and Cognition (STAC) is a conceptual, dynamic model that attempts to explain different cognitive performance levels, which are caused by individual adverse and compensatory neuronal processes. Adverse age effects (neuronal degradation) are based on neuronal challenges (structural changes such as atrophy) and functional degradation (e.g., reduced specificity). They determine the cognitive functional level in interaction with advantageous processes (compensatory scaffolding), which try to counteract degradation. This includes plasticity, for example by recruiting additional neuronal networks (Park and Reuter-Lorenz, 2009). The original model was extended by a revised version (STAC-r), which considers two additional influencing factors. The compensatory scaffold can be improved by interventions such as a healthy lifestyle (accumulation of neuronal resources) or worsened by negative influences such as stress or the APOE $\epsilon 4$ allele (degradation of neuronal resources) (Reuter-Lorenz and Park, 2014). Lifestyle factors play not only a role in the STAC-r model, but also in the Adaptive Capacity Model. Raichlen and Alexander (2017) proposed the Adaptive Capacity Model explaining the positive benefits of cognitively and physically demanding exercise: The brain responds to the combination of aerobic activity with control of motor systems, spatial navigation and memory, executive functions, and the control of sensory and attentional systems with a neuroplastic adaptive response by increasing capacity to lower energy costs. However, periods of inactivity lead to decreased structure and associated function, as reflected by age-related regional brain atrophy (Raichlen and Alexander, 2017). Conversely, no evidence can be found for a cognitive benefit (attention, memory, perception, executive functions, cognitive inhibition, cognitive speed, and motor function) of improved cardiorespiratory fitness in healthy older adults (Young et al., 2015). Only when combined with challenging cognitive exercise (e.g., dual task training) can positive effects on cognitive performance be observed (Gheysen et al., 2018). Lövdén et al. (2017, p. 473) categorize these frameworks and theoretical concepts explaining the influence of lifestyle factors in three dimensions: (a) through improving brain functioning and performance in younger age without altering brain aging per se, (b) by aiding compensatory reactions to primary brain aging, and (c) by fostering maintenance of a young-adults like brain in old age. However, the authors also state that we need to discover and measure what matters focusing on between-person differences in change.

Typically, scientists in the fields of cognitive aging, mild cognitive impairment and dementia focus on the description and explanation of age- and disease-related cognitive losses and the transition to dementia. However, a promising new approach focuses on the study of relatively healthy "optimal," "successful" or "exceptional" "high-performing older adults" and the question of which risk and protective factors are present in these physically and/or cognitively exceptional individuals (Gefen et al., 2014; Borelli et al., 2018). SuperAgers are defined as individuals aged 80 years or older with memory ability similar or superior to control participants 20-30 years younger. They show no signs of cortical atrophy relative to control participants (Harrison et al., 2012), their anterior cingulate cortex is significantly thicker (Gefen et al., 2014; Sun et al., 2016), and they show higher levels of physical activity (Bott et al., 2017). A recent study, examining different cognitive domains (memory, attention, language, visuospatial abilities) and lifestyle factors (physical and social activities, cognitively demanding activities, sleep, smoking, alcohol, stress) resulted in counter-intuitive results: superior cognition in older adults was associated with a busier and more stressful as well as socially isolated lifestyle in their midlives (Yu et al., 2019). The authors argue that longer exposure to physically and cognitively stimulating activities with a moderate stress level may serve to build up a larger capacity for neurocognitive scaffolding. On the other hand, the adherence to a higher number of lifestyle factors is associated with better cognitive function in later life (Clare et al., 2017; Weng et al., 2018). However, these lifestyle factors are typically measured at a single point in time, assessed via subjective self-reporting ratings, and tend to change over time (Mulder et al., 1998; Picavet et al., 2011). Overall, it is unclear if additional benefits of physical activity, fitness or meditation emerge from continued practice.

To our knowledge, no study has examined the effects of stable healthy lifestyle profiles on cognitive and motor performance. Therefore, the primary aim of this study was to determine whether there were differences in cognitive functions (working 
memory, inhibition, cognitive flexibility) between nuns/monks, master athletes and sedentary, but otherwise healthy older adults. Given the broad differences in lifestyle factors - such as BMI, physical activity, exercise, and fitness - of these three groups, we hypothesized that lifestyle stability in Master Athletes and nuns and monks will result in better cognitive performance. Additionally, we examined associations between demographic variables (education, sex, age), BMI, physical activity, exercise, and fitness and cognitive performance. We predicted stronger associations between fitness (as an expression of current exercise) and cognition than physical activity and exercise and cognition (Wouters et al., 2017).

\section{MATERIALS AND METHODS}

\section{Study Participants}

Sixty healthy older adults (23 men, 37 women) with no signs of dementia selected by the Montreal Cognitive Assessment (MoCA; score > 25; Nasreddine et al., 2005) were recruited from the southern part of Germany. The participants were divided into three groups reflecting different types and stability of lifestyle behaviors: (1) Nuns/Monks (NM; $n=20 ; 69-90$ years of age), (2) Master Athletes (MA; $n=20 ; 69-90$ years of age), and (3) community-dwelling older adults (CD; $n=20$; 68-91 years of age).

The nuns and monks lived in three different cloisters in the south of Germany (men: $n_{\text {total }} \sim 30, n \geq 65$ years $\sim 52 \%$; women: $n_{\text {total }} \sim 90, n \geq 65$ years $\sim 60 \%$ ), but - relative to the general population - order members are a homogenous population concerning a number of individual characteristics possibly relevant for health such as diet, activities of daily living, religiosity, and living arrangements (see also the German-Austria Cloister Study; Wiedemann et al., 2014). The master athletes in this study participated regularly in track and field competitions at European and World Master Athletics Championships, and were recruited through the German Track and Field Association (dlv). They exercise on average $4 \mathrm{~h}$ per week, had a track and field experience of at least 20 years and reported a balanced diet, and healthy sleep patterns. The group of community-dwelling older adults was mentally active (e.g., participation in hobbies such as craft, solving puzzles, playing an instrument, volunteering), but participated in little or no structured exercise. They were recruited through community newsletters and meetings places for older adults.

Inclusion criteria were age 65 years or more with normal or corrected-to-normal vision, and able to walk with or without any assistive device. Furthermore, individuals were screened for eligibility to participate based on their medical history. They were excluded if they were medically unstable based on self-report of the following: cardiopulmonary disease with symptoms such as shortness of breath or chest pain that would limit participation; current infectious, inflammatory or terminal conditions; prior neurological disease such as stroke or Parkinson's disease; acute illness, injury or pain at the time of testing that would limit testing performance. All participants were fluent German speakers.
All participants were informed of the nature and aim of the study, and signed a consent form. All procedures were in accordance to the Declaration of Helsinki with ethical standards, legal requirements and international norms. An internal ethics committee at the University of Stuttgart approved the study.

\section{Measures \\ Cognitive Assessment \\ $\mathrm{N}$-back task}

We used the N-back task introduced by Yun et al. (2010). All tasks were designed and presented by E-prime 2.02 (Psychology Software Tools, United States) on a $17^{\prime \prime}$ computer screen with a resolution of $1.920 \times 1200$ pixels. Participants had a distance of $\sim 70 \mathrm{~cm}$ from the screen, remained at this distance during the trials, and completed the $\mathrm{n}$-back tasks, starting with a 0 -back task followed by the 1-back and 2-back tasks in consecutive order.

Each trial consisted of a visual presentation of the letter a, b, c, or d (measuring $4 \times 4$ visual degrees; presented centrally on a monitor) for $500 \mathrm{~ms}$ followed by an interstimulus interval of $1500 \mathrm{~ms}$. During a n-back block, participants had to indicate via key-press whether, or not, the stimulus of the current trial matched the stimulus they saw N-steps back (see Figure 1). One half of the trials of a block were matches, that is, required participants to press the blue marked key ("L") as correct response, and one half of the trials were mismatches, that is, required participants to press the yellow marked key ("S") as correct response. The sequences of matches and mismatches were pseudo-randomly generated with the constraint that after a maximum of three matches at least one mismatch followed. On a regular PC keyboard, the buttons "L" and "S" were used and participants were instructed to leave their index fingers on the two buttons for the duration of the task.

Each subject was given written and verbal instructions on the task. Before starting the test, participants completed a practice run (one block of each condition). Subjects were instructed to perform the tasks to the best of their ability, answering as quickly as possible without sacrificing accuracy by pressing the corresponding key. Each of the three conditions consisted of 36 trials, with a 60-s inter-condition rest period.

The main dependent variables were reaction time (ms) and accuracy $\left(A^{\prime}\right)$. The percentages of hits (correct match) and false positives (incorrect match) for the n-back task were used to calculate $A^{\prime}$, a measure of detection sensitivity for each condition (Grier, 1971).

\section{Flanker task}

Inhibition was assessed by performance during a modified flanker task (Eriksen and Eriksen, 1974). The stimuli were presented using E-Prime software (Psychology Software Tools, Inc., Pittsburgh, PA) on a 17" computer monitor. Responses were registered using a standard QWERTZ keyboard. Participants sat approximately $70 \mathrm{~cm}$ away from the screen. During the task, participants attended to a centrally presented target stimulus (Chinese characters) amid an array of laterally presented flanking stimuli. During the compatible version of the task (all signs point

\footnotetext{
${ }^{2}$ http://www.pstnet.com/eprime.cfm
} 
in to the same direction: 不不不不不), participants were required to press "L." During the incompatible condition ( $\pi \cdot \pi x+\pi \cdot \pi)$, participants were required to press "S." Five letter stimuli, measuring $4.5 \mathrm{~cm}$ tall and separated by $1 \mathrm{~cm}$ were presented for $750 \mathrm{~ms}$ on a white background. Participants were instructed to identify the central sign as quickly as possible without sacrificing accuracy by pressing the corresponding key. A randomized inter-stimulus interval of 400-1200 ms was used, and both the number of trials within each condition and the frequency of target direction were equiprobable, with randomly presented trials within each task block. Participants were administered five blocks of 16 trials for each compatibility condition and given a brief break and encouragement between each block. For all analyses, individual trials with RT's outside the 200-1650 ms post-stimulus onset window and incorrect trials were excluded from the RT analysis (Wu et al., 2011). The main dependent variables were reaction time (ms) and accuracy (\%) for congruent and incongruent trials.

\section{Covariates}

All participants were invited to a face-to-face interview to answer standardized questionnaires. The questionnaire included socio-demographic characteristics (i.e., age, gender, education level, marital status, dwelling space), lifestyle habits (i.e., physical activity, exercise; PAQ-50+; Huy and Schneider, 2008), depressive symptoms [Geriatric Depression Scale (GDS); Yesavage et al., 1983], and past medical history (e.g., diabetes).

\section{General cognitive function}

The MoCA (Nasreddine et al., 2005) was used to assess cognitive function, which is designed as a rapid screening tool for mild cognitive dysfunction. It assesses various cognitive domains, including attention and concentration, executive function, memory, language, visuo-constructional skills, conceptual thinking, and recall. Scores on the MoCa can range between 0 and 30, with lower scores indicating worse global cognitive functioning. The MoCA is a reliable and valid estimate of overall global cognitive abilities with a cutoff of 24 as optimal for a diagnosis of Mild Cognitive Impairment (Nasreddine et al., 2005; Kenny et al., 2013).

\section{Depression}

We applied the 15-item GDS (Yesavage et al., 1983), a self-report assessment to evaluate the degree of depressive symptoms of participants. The scores range from 0 to 15 points, with a higher score indicating a more depressive state. GDS scores of $0-5$ were classified as having no depression, scores of 6-10 were classified as slight depression, and scores of 11-15 were classified as moderate to severe depression.

\section{Physical activity and exercise}

Everyday physical activity was assessed using the Physical Activity Questionnaire for the population aged 50 years and older (PAQ-50+, Huy and Schneider, 2008). The 37 questions are designed to estimate the physical activity of older adults based on different activities, including sport, housework, yard work, job, and leisure activities. Each activity can be evaluated by MET-values or the duration (min/week). The energy expenditure (kcal per week) can additionally be calculated by an equation from the specific activity/MET value, the duration of the activity performance and the weight of the person. The sum of all activities corresponds to the total result of the PAQ $50+$ in kilocalories or the amount of time per week.

Subjects were also evaluated on their sports biography: participants were asked in which organized activities (participation through a formal club, max. three different activities) they had participated over the past 12 months (see also Schott et al., 2016). Next, they were asked how many days a week, and minutes per session, they had participated in that particular activity. Total exercise duration (h/week) was calculated as follows: (frequency $1 \times$ duration 1 ) + (frequency $2 \times$ duration $2)+($ frequency $3 \times$ duration 3$)$.

\section{Functional fitness}

Physical performance was evaluated by two tests out of the Senior Fitness Test (SFT), which was developed for early identification of older adults who are at risk of losing functionality (Rikli and Jones, 2013): (1) 30s chair stand (assessment of lower body strength, repetitions within 30s), and (2) 30s arm curl (assessment of upper body strength, repetitions within 30s). The present study has followed the testing procedures suggested by the SFT manual. The 30s chair stand test consisted of recording the number of repetitions of rising from a chair and return to the seated position, as fast as possible, with their arms folded across their chest. The 30 s arm curl consisted of recording the number of repetitions of biceps curls that can be completed in 30s, holding a hand weight of $2 \mathrm{~kg}$ for women and $3 \mathrm{~kg}$ for men.

\section{Body composition}

Height and weight were recorded using a standard protocol. Body mass index was calculated as weight in kilograms divided by height in meters squared $\left(\mathrm{kg} / \mathrm{m}^{2}\right)$.

\section{Procedure}

Data was collected from May to September 2013. The questionnaires, the fitness and cognitive testing were administered individually during a single testing session lasting approximately $2 \mathrm{~h}$, which included breaks as needed. All tests were administered by the same trained specialist (M.Sc. in Gerontology) with standardized procedures including standard instruction and practice. Additionally, the participants were encouraged to ask questions, if needed, throughout the procedures for better understanding and compliance.

\section{Statistical Analysis}

Statistical analyses were implemented on SPSS v.25 (SPSS, Chicago, IL). We first explored dependent variables to examine missing data points, normality of distributions, and presence of outliers. The Kolmogorov-Smirnov tests indicated that all variables (except exercise, GDS, RTs) entered into the analysis were normally distributed.

Potential baseline group differences for continuous variables (i.e., age, height, weight, BMI, physical activity, education, fitness) were assessed using ANOVAs, and categorical demographic variables (i.e., gender) were compared by chi-square test. 
Responses [accuracy $\left.\left(\%, A^{\prime}\right)\right]$ and reaction times of the n-back and/or flanker stimulus were recorded in every trial. Trials with reaction times longer or shorter than the median of all reaction times of a participant \pm 3 times the median absolute deviation were excluded from further analysis, as suggested by Leys et al. (2013). Median RTs for correct trials were then calculated for each condition as an indicator of processing efficiency. Flanker RTs and accuracy rates were submitted to ANCOVAs with repeated measures including the within-subjects factors cognitive load (block 1-5), congruency (congruent vs. incongruent) as well as the between-subjects factor of group (Master Athletes vs. Nuns/Monks vs. sedentary older adults) controlled for sex (due to the unequal distribution of men and women in our subsamples). n-back sensitivity $\left(A^{\prime}\right)$ and RT were compared similarly with cognitive load (0-back, 1-back, 2-back) as within-subjects factor and group as between-subjects factor controlled for sex. Analyses with three or more within-subjects levels report $p$-values after Greenhouse-Geisser correction for violations of sphericity. Significance levels were set at $p=0.05$, and post hoc comparisons were conducted using Bonferroni correction. Partial eta-square $\left(\eta_{\mathrm{p}}^{2}\right)$ is reported to indicate effect size. Results of these analyses indicate whether, when inhibition and working memory were considered separately, there were significant differences on RT and error measures associated with group, cognitive load, and the interaction of group and cognitive load.

Pearson correlation analysis was employed to examine associations among all relevant variables. The independent variables with low explanatory power were not included in the regression analysis. Separate hierarchical stepwise regression analyses with orthogonal contrast coding were employed in order to examine whether differences between lifestyle groups remained when controlling for other relevant characteristics that might confound the association between lifestyle and cognitive functions. The first contrast compared Master Athletes and Nuns/Monks [-1] to the sedentary group [+2] (sed_active). The second contrast compared Master Athletes [-1] and Nuns/Monks [+1] (MA_NM). To address potential multicollinearity, the independent variables as well as the interaction terms were mean centered (Aiken and West, 1991). In the first block, the dependent variables from the n-back and the Flanker-task (accuracy values and RTs) were regressed on age, sex, and education. In block 2 , the two group contrasts (sed_active, MA_NM), and in block 3, fitness, exercise, and physical activity were regressed on cognitive performance. Finally, in the fourth block, the corresponding product terms between group contrasts and fitness, exercise, and physical activity were entered.

\section{RESULTS}

\section{Participant Characteristics}

A description of the three groups is given in Table 1. The three groups did not differ significantly in terms of age, years of education, general cognition (MoCA), and depression (GDS). The percentage of men and women was significantly different across the three groups. The proportion of male subjects was higher in the group of the Master Athletes than in groups of sedentary older adults $(p=0.057)$ and nuns and monks $(p=0.025)$. Additionally, Master Athletes had a lower BMI, took less medication, performed better on the 30s-Chair Rise test and the 30s-Arm Curl test, and exercised longer compared to the sedentary groups as well as the groups of nuns and monks.

\section{Working Memory Performance}

Detection sensitivity scores $\left(A^{\prime}\right)$ and median reaction times for sedentary older adults, monks/nuns, and master athletes are presented in Figure 2. The manipulation of cognitive load was successful as indicated by a main effect of load, $F(1.51,80.2)=7.54, p<0.001, \eta_{\mathrm{p}}^{2}=0.125$, with lower overall detection sensitivity in the 2-back $(M=0.714$; $\mathrm{SE}=0.016)$, than in the 1-back $(M=0.811 ; \mathrm{SE}=0.028)$, and the 0 -back task $(M=0.979 ; \mathrm{SE}=0.003)$. The 3 (cognitive load) $\times 3$ (group) interaction yielded also a significant effect, $F(3.03,80.2)=2.74$, $p=0.048, \eta_{\mathrm{p}}^{2}=0.094$. Post hoc tests revealed that sensitivity was especially better in nuns/monks compared to sedentary older adults. In addition, there was a significant load $\times$ sex interaction, $F(1.51,80.2)=7.39, p=0.002, \eta_{p}^{2}=0.122$. Further analysis revealed no differences between men and women in the 0 -back condition, but higher accuracy rates for women in the 1-back condition as well as higher accuracy rates for men in the 2-back condition.

The 3 (cognitive load) $\times 3$ (group) repeated measures ANCOVA on median RTs controlled for sex revealed a load by group interaction, $F(3.47,91.8)=6.15, p<0.001, \eta_{\mathrm{p}}^{2}=0.188$ with RTs slower for tasks with a higher load in the sedentary older adults, but faster RTs in the 2-back condition compared to the 1 -back condition in nuns/monks, and master athletes. No main effects for cognitive load or the interaction load $\times$ sex were found.

\section{Inhibitory Control}

Accuracy scores and median reaction times for sedentary older adults, monks/nuns, and master athletes are presented in Figure 3. For the percentage of correct responses, the 5 (cognitive load) $\times 2$ (congruency) $\times 3$ (group) repeated measures ANCOVA controlled for sex resulted in a significant interaction effect of congruency $\times$ group, $F(2,56)=7.43$; $p=0.001 ; \eta_{\mathrm{p}}^{2}=0.210$. Post hoc tests revealed the largest flanker interference for sedentary older adults (congruent $86.7 \% \pm 1.77$ vs. incongruent $72.1 \% \pm 2.84$ ) compared to the master athletes (congruent $91.3 \% \pm 1.83$ vs. incongruent $86.8 \% \pm 2.93$ ) and the nuns/monks (congruent $98.1 \% \pm 1.79$ vs. incongruent $94.9 \% \pm 2.87$ ). The main effects of load and congruency as well as the interactions load $\times$ group, load $\times$ sex, congruency $\times$ sex, load $\times$ congruency $\times$ group interactions were not significant.

To analyze performance of RTs in the flanker task, a 5 (cognitive load) $\times 2$ (congruency) $\times 3$ (group) repeated measures ANCOVA controlled for sex was conducted. Results revealed a main effect for congruency, $F(1,56)=8.39 ; p=0.005$; $\eta_{\mathrm{p}}^{2}=0.130$, showing faster RTs on congruent (1032 ms) than in incongruent trials $(1132 \mathrm{~ms})$. There was also a significant congruency $\times$ group interaction, $F(2,56)=5.48 ; p=0.007$; $\eta_{\mathrm{p}}^{2}=0.164$. Post hoc tests revealed the largest flanker interference for sedentary older adults (congruent $1122 \mathrm{~ms} \pm 56.7$ vs. 
TABLE 1 | Participant characteristics by group.

\begin{tabular}{|c|c|c|c|c|}
\hline & $\begin{array}{l}\text { Sedentary } \\
(n=20)\end{array}$ & $\begin{array}{l}\text { Nuns/Monks } \\
(n=20)\end{array}$ & $\begin{array}{l}\text { Master Athletes } \\
\qquad(n=20)\end{array}$ & Statistical analysis \\
\hline Age (years) & $76.4 \pm 5.96$ & $77.5 \pm 5.56$ & $76.5 \pm 5.33$ & $F(2,57)=0.23, p=0.792, \eta^{2}=0.008$ \\
\hline $\mathrm{BMI}\left(\mathrm{kg} / \mathrm{m}^{2}\right)$ & $25.7 \pm 3.02$ & $26.3 \pm 4.09$ & $23.5 \pm 3.32^{\#}$ & $F(2,57)=3.51, p=0.036, \eta^{2}=0.110$ \\
\hline Medication (n) & $0.75 \pm 0.85$ & $0.65 \pm 0.75$ & $0.15 \pm 0.37^{*}$ & $F(2,57)=4.39, p=0.017, \eta^{2}=0.133$ \\
\hline Education (years) & $11.1 \pm 2.96$ & $11.9 \pm 3.57$ & $11.8 \pm 3.04$ & $F(2,57)=0.37, p=0.692, \eta^{2}=0.013$ \\
\hline 30s-Chair Rise test (n) & $15.4 \pm 4.03$ & $16.5 \pm 4.54$ & $22.0 \pm 3.99^{\# \# \#, * * *}$ & $F(2,57)=14.2, p<0.001, \eta^{2}=0.333$ \\
\hline 30s-Arm Curl test (n) & $18.8 \pm 4.29$ & $19.7 \pm 4.02$ & $28.0 \pm 3.97^{\# \# \#, * * *}$ & $F(2,57)=30.5, p<0.001, \eta^{2}=0.517$ \\
\hline PAQ (h/week) & $19.0 \pm 8.47$ & $42.6 \pm 14.3^{* * *}$ & $26.4 \pm 16.7^{\# \# \#}$ & $F(2,57)=15.8, p<0.001, \eta^{2}=0.356$ \\
\hline Exercise (min/week) & $42.0 \pm 64.1$ & $37.5 \pm 72.1$ & $243.0 \pm 109.8^{\# \# \#, * * *}$ & $F(2,57)=38.7, p<0.001, \eta^{2}=0.576$ \\
\hline
\end{tabular}

Data are presented as mean \pm standard deviation. Differences were evaluated by the Chi-square test or analysis of variance, followed by the Bonferroni test. BMI, body mass index; MoCA, Montreal Cognitive Assessment; GDS, Geriatric Depression Scale; PAQ, Physical Activity Questionnaire. ${ }^{*} p<0.05$ and ${ }^{* * *} p<0.001$ compared with the sedentary group. ${ }^{\#} p<0.05$ and $\# \#$ $p<0.001$ compared with the nuns/monks.

\section{0-Back: \\ 1-Back: Is the letter}

Is the letter an „a“? the same as before?
2-Back: Is the letter the same as two before?
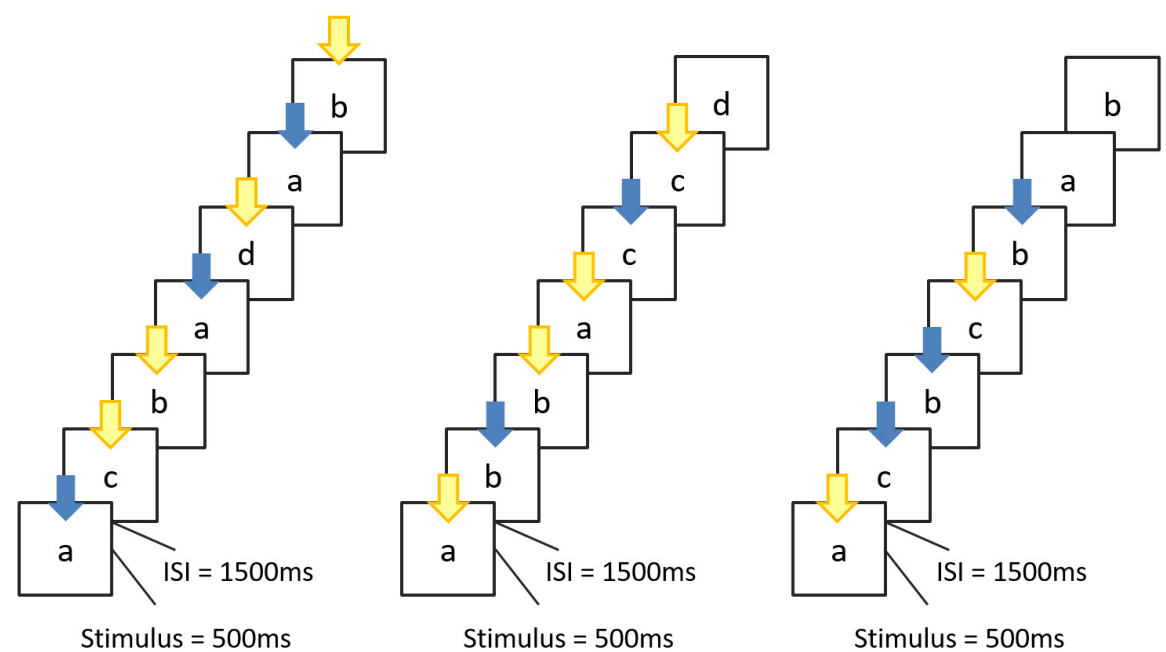

Stimulus $=500 \mathrm{~ms}$

FIGURE 1 | Schematic overview of the n-back task. Stimulus presentation was $500 \mathrm{~ms}$, interstimulus interval (ISI) $1500 \mathrm{~ms}$. During the ISI, a fixation cross was displayed. Participants were allowed to respond until the next stimulus appeared. In the 0-back condition, participants responded to the single pre-specified target letter "a"; in the 1-back condition, the target was any letter identical to the one immediately preceding it (i.e., one trial back); and in the 2-back condition, the target was any letter that was identical to the one presented two trials back. In this manner, the WM load (storage and manipulation demands) increased incrementally from the 0 -back to the 2-back task. The color of the arrow refer to the button to be pressed (blue = match; yellow = mismatch).

incongruent $1259 \mathrm{~ms} \pm 64.5)$ compared to the nuns/monks (congruent $1044 \mathrm{~ms} \pm 55.1 \mathrm{vs.}$ incongruent $1168 \mathrm{~ms} \pm 65.1$ ) and the master athletes (congruent $929 \mathrm{~ms} \pm 56.3$ vs. incongruent $968 \mathrm{~ms} \pm 66.5)$. The block $\times$ congruency $\times$ group interaction approached significance, $F(6.32,177)=1.86 ; p=0.087 ; \eta_{\mathrm{p}}^{2}=0.062$ (see also Figure 3).

\section{Relationship Between Demographic Variables, Fitness, Physical Activity, and Cognitive Performance}

Correlations among variables in the overall sample are reported in Table 2. Accuracy/sensitivity of working memory as well as inhibitory performance is positively associated with physical activity, while RTs are associated with age, sex, fitness, and exercise.

Results for the hierarchical linear regression analyses are presented in Table 3. These analyses were conducted in order to examine if fitness, exercise, and physical activity explained a larger portion of variance than group (i.e., nuns/monks vs. Master Athletes vs. sedentary controls) in measures of working memory and inhibition irrespective of age, sex, and education. The contrast sed_active, which reflected our hypothesized cognitive performance pattern (sedentary controls $<$ nons/monks and master athletes) reached significance in all variables, but the RTs 

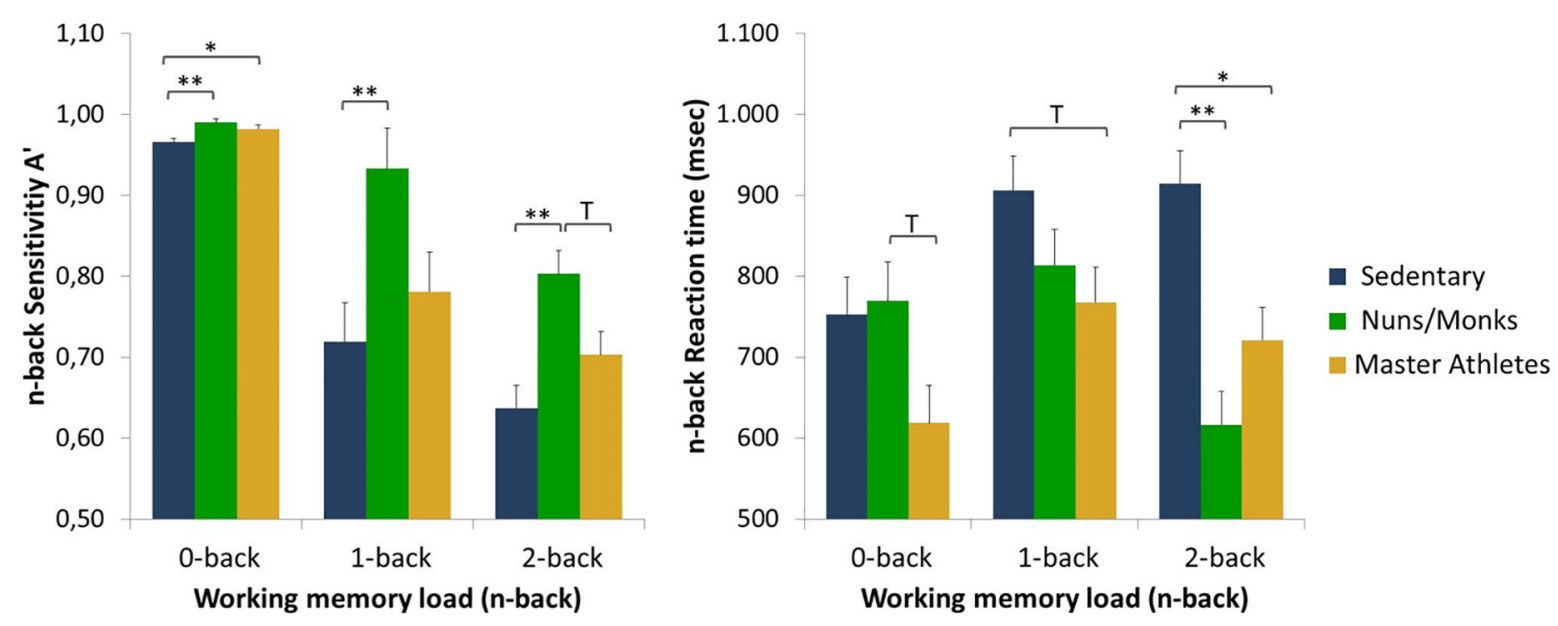

FIGURE 2 | Detection sensitivity $\left(\mathrm{A}^{\prime}\right)$ and reaction times as a function of working memory load (0-back, 1-back, and 2-back) and group (sedentary older adults, monks and nuns, master athletes). Bar graphs show sex adjusted mean $\pm \mathrm{SEM}$; ${ }^{*} p<0.05,{ }^{* *} p<0.01$, and T $p<0.10$.

for the congruent Flanker task. The alternate orthogonal contrast MA_NM reached significance for the composite sensitivity score for n-back, the accuracy rates for the congruent Flanker task as well as the RTs for the incongruent Flanker task, indicating better performance in accuracy measures in nuns/monks compared to master athletes. However, master athletes outperformed nuns/monks in RTs. Only for the RTs in the congruent condition of the Flanker task could we show an additive effect of fitness with higher-fit individuals showing faster RTs than low-fit individuals.

\section{DISCUSSION}

A significant amount of studies has shown that there is an association between cognitive performance and lifestyle behaviors such as physical activity, fitness, sleep, nutrition, and spirituality in older adults (Clare et al., 2017). However, these studies usually included individuals who were not particularly inclined to exhibit these behaviors at all or were shown to adhere rather irregularly. Here, we report for the first time the relationship of stable, long-term lifestyles as can be found for Master Athletes and nuns/monks with cognitive performances. In the present study, we find that nuns/monks outperform Master Athletes as well as sedentary controls in accuracy values of working memory and inhibitory performance, while Master Athletes show faster reaction times compared to the other two groups. In addition, we hypothesized that higher levels of physical activity, exercise, and fitness would be associated with better cognitive performance. The data from our study partially supports this expectation. We found that fitness (strength of upper and lower extremities) and exercise were associated with faster reaction times (but not accuracy rates) on the n-back- and the Flanker Task. This indicates that older adults with a higher overall strength and longer durations of structured exercise produced faster reaction times. In contrast, greater physical activity levels were only correlated with accuracy rates of the n-back- and the Flanker Task.

\section{Determinants of Health Status in Nuns/Monks, Master Athletes, and Sedentary Controls}

Elevated BMI may be associated with higher risk of dementia (Boyle et al., 2017). When compared to the Schienkiewitz et al. (2017) BMI data, it was apparent that Master Athletes (75\%) and sedentary controls (50\%), but not nuns/monks (40\%) have a higher proportion of individuals with a BMI indicative of underweight or normal compared to the general German population in this age group (40\%). Such results were similar with previous research that demonstrated a healthy BMI in Master Athletes (Fien et al., 2017) and nuns/monks (Bennett et al., 2018). Another essential aspect of preserving health and function in older adults is the ability to maintain muscle mass and power. All of the Master Athletes, 75\% of the nuns/monks, and $60 \%$ of the sedentary controls in our study performed above criterion-referenced fitness standard for the 30s Arm Curl test and the 30s Chair Rise test (Rikli and Jones, 2013). Inspection of other health-related indicated that only $1.7 \%$ of our sample took three medications per day, and 3.3\% had a GDS score of $5.40 \%$ of the sedentary controls and $30 \%$ of the nuns/monks are engaged in exercise once or twice a week for at least $30 \mathrm{~min}$; Master Athletes exercise twice $(45 \%)$, three $(35 \%)$ or five times $(20 \%)$ a week for at least $60 \mathrm{~min}$, which is similar to data reported by Fien et al. (2017). Eighty-five percent of the group of nuns/monks report daily leisure or gardening activities for at least $30 \mathrm{~min}$ each day; Master Athletes as well as sedentary controls are less active with $65 \%$, respectively $50 \%$. These moderate to vigorous levels of exercise and physical activities are also related to mortality: As shown in a previous study, Polish Olympic athletes experienced a slower rate of aging, a lower risk of mortality as well as a longer life-expectancy compared to nuns/monks and actors 


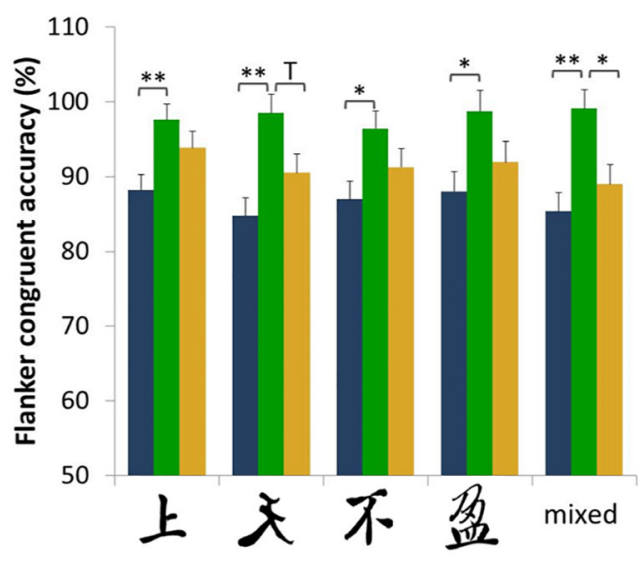

inhibition load

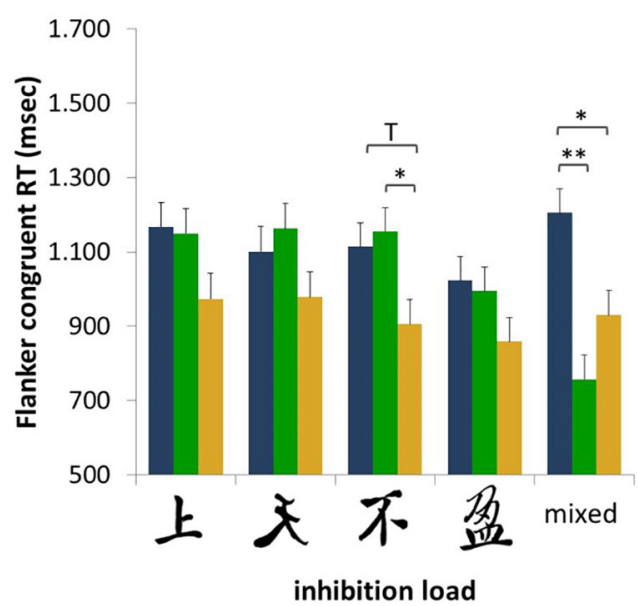

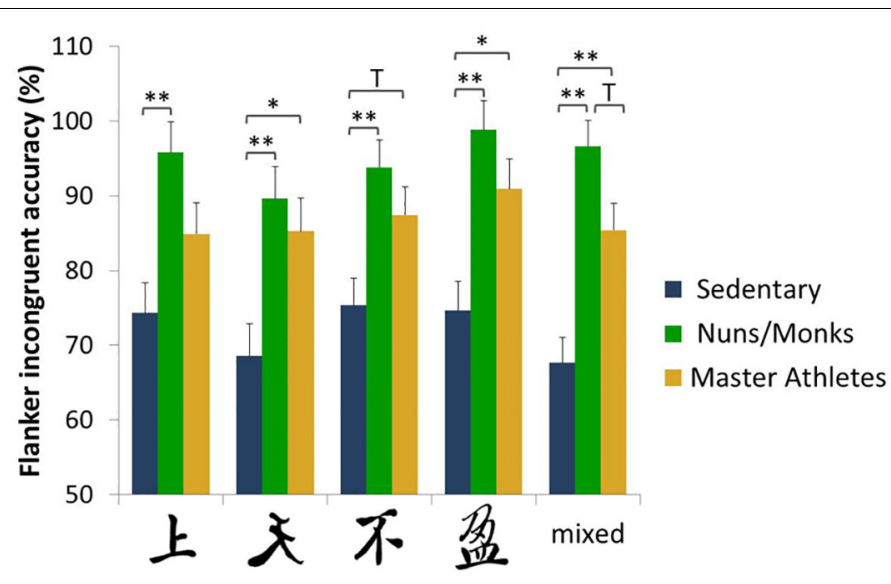

inhibition load

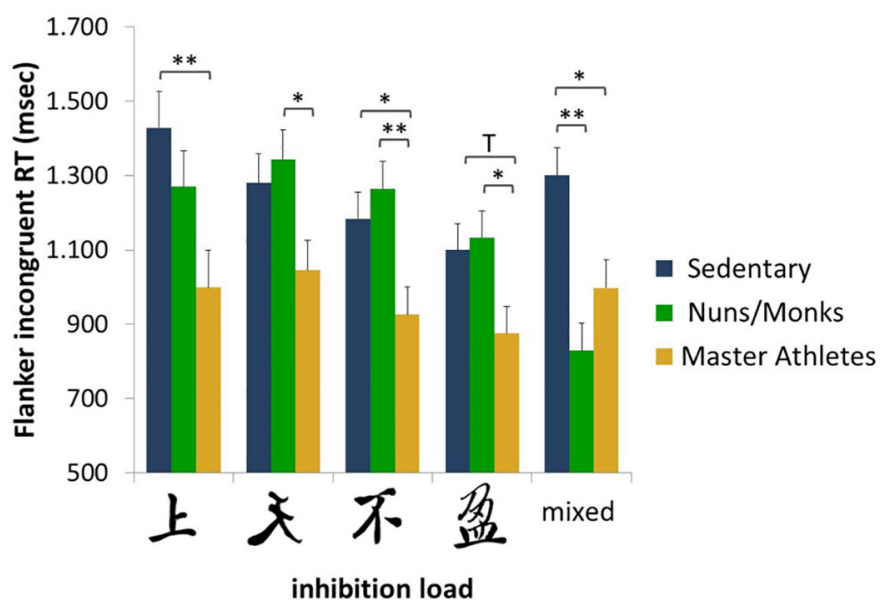

FIGURE 3 | Accuracy rates and reaction times for congruent and incongruent trials as a function of inhibition load and group (sedentary older adults, monks and nuns, master athletes). Bar graphs show sex adjusted mean $\pm \mathrm{SEM} ;{ }^{*} p<0.05,{ }^{* *} p<0.01$, and T $p<0.10$.

TABLE 2 | Correlations between working memory and inhibition with demographic, fitness, and physical activity variables for all participants $(n=60)$.

\begin{tabular}{|c|c|c|c|c|c|c|}
\hline & $\begin{array}{c}\text { n-back } A^{\prime} \\
\text { comp }\end{array}$ & $\begin{array}{l}\text { n-back RT } \\
\text { comp }\end{array}$ & $\begin{array}{l}\text { Flanker congr } \\
\text { ACC comp }\end{array}$ & $\begin{array}{c}\text { Flanker } \\
\text { incongr ACC } \\
\text { comp }\end{array}$ & $\begin{array}{l}\text { Flanker congr } \\
\text { RT comp }\end{array}$ & $\begin{array}{c}\text { Flanker } \\
\text { incongr RT } \\
\text { omp }\end{array}$ \\
\hline Age (years) & 0.16 & $0.32^{*}$ & -0.24 & -0.10 & $0.32^{*}$ & $0.28^{*}$ \\
\hline Sex & 0.18 & $0.30 *$ & -0.03 & -0.02 & $0.33^{*}$ & $0.29^{*}$ \\
\hline BMI $\left(\mathrm{kg} / \mathrm{m}^{2}\right)$ & -0.15 & 0.18 & 0.06 & 0.04 & 0.03 & 0.12 \\
\hline Medication (n) & -0.10 & $0.33^{*}$ & 0.05 & -0.07 & 0.17 & 0.22 \\
\hline GDS & 0.14 & 0.13 & -0.23 & -0.14 & 0.17 & 0.19 \\
\hline 30s-Chair Rise test (n) & -0.05 & $-0.45^{* *}$ & 0.07 & 0.10 & $-0.45^{* *}$ & $-0.44^{* *}$ \\
\hline 30s-Arm Curl test (n) & -0.01 & $-0.49^{* *}$ & 0.18 & 0.23 & $-0.52^{* *}$ & $-0.57^{* *}$ \\
\hline PAQ (h/week) & $0.32^{*}$ & -0.25 & $0.37^{* *}$ & $0.48^{* *}$ & -0.11 & -0.09 \\
\hline Exercise (min/week) & -0.09 & $-0.31^{*}$ & 0.05 & 0.08 & $-0.39^{* *}$ & $-0.40^{* *}$ \\
\hline
\end{tabular}

Bolded values in black indicate statistically significant correlations ( $p<0.05)$; comp, composite score; ACC, accuracy; congr, congruent; incongr, incongruent. 
TABLE 3 | Summary of separate hierarchical linear regression analysis for the interaction between demographic variables, orthogonal contrasts, fitness, exercise, and physical activity for the composite scores of the cognitive performance (only results for final models are presented).

\begin{tabular}{|c|c|c|c|c|c|c|}
\hline Dependent variable & Predictor & $B$ & SE $B$ & $\beta$ & $\Delta R^{2}$ & $R^{2}$ \\
\hline \multirow[t]{2}{*}{ n-back $A^{\prime}$} & sed_active & -0.77 & 0.173 & $-0.490^{* *}$ & 0.240 & 0.279 \\
\hline & MA_NM & 0.68 & 0.299 & $0.251^{*}$ & 0.063 & \\
\hline \multirow[t]{3}{*}{ n-back RT } & Age & 0.14 & 0.04 & $0.344^{* *}$ & 0.102 & 0.353 \\
\hline & Sex & 1.15 & 0.50 & $0.245^{*}$ & 0.089 & \\
\hline & sed_active & 0.72 & 0.17 & $0.445^{* *}$ & 0.195 & \\
\hline \multirow[t]{2}{*}{ Flanker congr ACC } & sed_active & -1.18 & 0.31 & $-0.435^{* *}$ & 0.190 & 0.247 \\
\hline & MA_NM & 1.35 & 0.53 & $0.287^{*}$ & 0.082 & \\
\hline \multirow[t]{3}{*}{ Flanker in-congr ACC } & Education & 0.25 & 0.13 & $0.202^{*}$ & 0.071 & 0.385 \\
\hline & sed_active & -1.55 & 0.29 & $-0.552^{* *}$ & 0.301 & \\
\hline & MA_NM & 1.02 & 0.50 & $0.211^{*}$ & 0.044 & \\
\hline \multirow[t]{4}{*}{ Flanker congr RT } & Sex & 1.47 & 1.09 & 0.173 & 0.108 & 0.276 \\
\hline & Age & 0.17 & 0.09 & $0.224^{\top}$ & 0.106 & \\
\hline & sed_active & 0.39 & 0.37 & 0.132 & 0.062 & \\
\hline & Fitness & -0.13 & 0.06 & $-0.309^{*}$ & 0.050 & \\
\hline \multirow[t]{4}{*}{ Flanker in-congr RT } & Sex & 1.53 & 1.00 & 0.183 & 0.082 & 0.242 \\
\hline & Age & 0.21 & 0.08 & $0.279^{*}$ & 0.080 & \\
\hline & sed_active & 0.85 & 0.33 & $0.294^{*}$ & 0.080 & \\
\hline & MA_NM & 1.19 & 0.60 & $0.239 *$ & 0.052 & \\
\hline
\end{tabular}

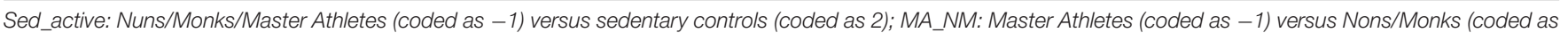
1) vs. sedentary controls (coded as 0 ); ${ }^{\top} p<0.10,{ }^{*} p<0.05$, ${ }^{* *} p<0.01$; ACC, accuracy; congr, congruent; incongr, incongruent.

(Gajewski and Poznańska, 2008). Overall, our data suggests that we examined a functional and healthy group of older adults (Ravaglia et al., 2008).

\section{Cognitive Performance}

As expected and in accordance with past research, that have demonstrated that older adults with higher fitness levels show better performances specifically in the executive domains (Taran et al., 2013; Thomas et al., 2013; Tseng et al., 2013; Alfini et al., 2016; Zhao et al., 2016; Dupuy et al., 2018), the Master Athletes in our study outperformed sedentary controls in both working memory performance as well inhibitory control (for reaction times, but not for accuracy). Our results are in line with Dupuy et al. (2018), who found in high-fit older adults faster reaction times for a Stroop task as a measure of inhibitory control. Similarly, our results align with Zhao et al. (2016) who reported better performances on a verbal memory task and a reaction time test [both from the Immediate Post-concussion Assessment and Cognitive Testing (ImPACT) tool] in Master Athletes compared to sedentary control. Also, Taran et al. (2013) showed significantly better performance on verbal learning and memory tasks (Rey Auditory Verbal Learning Test) as well as faster processing speed (Trail Making Test). In contrast, Wouters et al. (2017) found a positive relationship between gait speed as marker of fitness and working memory, but were not able to confirm a positive association between lifelong physical activity and working memory or executive function. Competitive cognitive engagement may be associated with better structural and functional connectivity across the human brain as expertise and the training of motor skills are often associated with changes in brain structure and function (Dayan and Cohen, 2011; Binder et al., 2017;
Godde and Voelcker-Rehage, 2017; Gheysen et al., 2018). Indeed, Binder et al. (2017) found that a multi-domain motorcognitive training improves neural lateralization and functional connectivity in older adults. Also, Tseng et al. (2013) reported that life-long exercise of Master Athletes resulted in higher gray and white matter concentrations in the subgyral, cuneus, and precuneus regions compared to sedentary controls; area which are related to visuospatial function, motor control, and working memory.

The group of nuns and monks represent a group of relatively homogenous individuals (e.g., income, occupation, and religiosity) with similar physical and socioeconomic characteristics of their communities. The rules of order life determine many health behaviors, which might be an effective lifestyle to increase brain- and cognitive-reserve capacity (e.g., low alcohol and tobacco consumption, daily prayer and meditation; Bowen and Luy, 2018). In general, nuns/monks outperformed Master Athletes and sedentary controls in both working memory performance as well inhibitory control (for accuracy) consistent with various attention related advantages that have been documented in older adults with meditation training (see for an excellent overview Christie et al., 2017). van Leeuwen et al. (2012) argue that meditation (similar to praying) improves the allocation of attention in space and the ability to adjust the focus of attention from a global pattern to the fine grained detail of an image (here Chinese characters). However, one has to take into account that meditation addresses the "self," while spiritual practices focus on internal and external sense of connection to a higher entity (Barnby et al., 2015). To the best of our knowledge, studies comparing executive function of nuns and monks with community-dwelling older adults are 
non-existent. Larger studies as the "Religious Order Study" or the "Nuns Study" focuses on the examination of Alzheimer Disease pathology. In a recent attempt to describe a neurophysiological model of religious experiences, Newberg (2017) suggests a number of complex interactions of the prefrontal cortex (sustained attention) with the thalamus (alteration in the sense of realness and clarity), posterior superior parietal lobe (overall sense of self), limbic system (modulation or cortical arousal), and autonomic nervous system (relaxation, quiescence). In addition, he highlights the role of neurotransmitters (e.g., dopamine, serotonin, and acetylcholine). Since the frontal lobe is one of the brain regions most affected by aging processes, this shows the potential of meditative exercises in maintaining cognitive health in older adults (Christie et al., 2017).

Studies on the relationship of lifestyle factors and cognition have promoted the development of theoretical models that assess and attempt to identify the different biological, neurological, and psychological pathways by which e.g., fitness, exercise, intellectual engagement, meditation, sleep, and diet improve cognitive performance (especially cognitive control) such as the STAC-r (Reuter-Lorenz and Park, 2014), Cognitive Reserve Stern (2002), or the Adaptive Capacity Model (Raichlen and Alexander, 2017). The potential mechanisms for improved cognitive performance can be divided into three categories: neurobiological (e.g., neurotrophin gene; gray matter volume and activation; upregulation of neurotransmitters); psychosocial (e.g., self-efficacy, social connectedness, mood and emotions), and behavioral (e.g., quality of sleep, coping, selfregulation skills). For example, Raichlen and Alexander (2017) propose in accordance with the neurobiological hypothesis that energy saving mechanisms in response to reduced physical and cognitive activity would affect neurogenesis and synaptogenesis leading to reduced gray matter and regional brain atrophy. However, tolerance to neuropathological changes is attributed not only to neurobiological but also to psycho-social factors (Arenaza-Urquijo et al., 2015; Christie et al., 2017; Wilson and Bennett, 2017). Several studies found, that frequent participation in social activities are related with better cognitive performance, but especially emotional support and self-efficacy (beliefs about the ability to complete a task or achieve a goal) may be stronger associated with cognition than other psycho-social factors (Zahodne et al., 2014). Master Athletes exhibit not only higher levels of self-efficacy (Dionigi et al., 2011), but are also considered as exemplars of high social functioning (Geard et al., 2017).

Although our study has revealed intriguing novel findings, several limitations are worthy of note. First, we cannot conclude a causal link between lifestyle stability and cognitive performance, as this would require a longitudinal design. Indeed, the level of cognitive ability might influence the maintenance of physical activity or the cessation of smoking, as much as these habits might influence cognitive performance. In addition, due to our design, we cannot make any assumptions about cognitive function over time, since other genetic or environmental factors as well as other lifestyle behaviors (e.g., diet, smoking, alcohol consumption) may be involved, which were not included in our study (e.g., dietary and sleep patterns, smoking, alcohol consumption). Lifestyle behaviors, such as physical activity were self-reported, which may have led to recall bias. This could have underestimated the relationship between cognition and physical activity. Another limitation of the current study involves the lack of additional fitness measures (beyond strength tests), such as aerobic fitness $\left(\mathrm{VO}_{2} \mathrm{max}\right)$. Lastly, this study was based on a small, but unique sample size. Thus, the results must be interpreted with caution. However, the rules of a monastery as well as the rules of the successful participation in a competition require the constant observance of certain health-related behavioral patterns (Geard et al., 2017; Bowen and Luy, 2018).

\section{CONCLUSION}

The primary novel results from this cross-sectional study with 20 nuns/monks, 20 Master Athletes, and 20 sedentary controls were that first, nuns/monks demonstrated better accurate cognitive performance than Master Athletes, while Master Athletes exhibited faster responses than both other groups. Second, fitness was associated with inhibitory control, and physical activity with the most demanding working memory task. Third, group was a statistically significant predictor for accurate performance, but not for reaction times. Our results on lifetime engagement in physical activity (Master Athletes) as well as "meditative exercises" (prayers, nuns/monks) seem to support the efficacy of stable and healthy lifestyles in preserving cognitive functioning. Although, we still have to speculate about why there is variance we cannot account for between our three groups (Lövdén et al., 2017). In the future, a key objective is to identify and describe the complex interaction of meaningful genetic, biological and environmental predictors, especially as they distinguish between exceptional groups and normal or groups with motor and/or cognitive impairment (Dixon and Lachman, 2019). Longitudinal studies are recommended to further examine the causal relationship of lifestyle stability and cognitive function in such specific cohorts.

\section{ETHICS STATEMENT}

All participants were informed of the nature and aim of the study, and signed a consent form. All procedures were in accordance to the Declaration of Helsinki with ethical standards, legal requirements and international norms. An internal ethics committee at the University of Stuttgart approved the study.

\section{AUTHOR CONTRIBUTIONS}

NS conceived the experiment and analyzed the data. KK collected the data. NS and KK wrote the manuscript. 


\section{REFERENCES}

Aiken, L. S., and West, S. G. (1991). Multiple Regression: Testing and Interpreting Interactions. London: Sage.

Alfini, A. J., Weiss, L. R., Leitner, B. P., Smith, T. J., Hagberg, J. M., and Smith, J. C. (2016). Hippocampal and cerebral blood flow after exercise cessation in master athletes. Front. Aging Neurosci. 8:184. doi: 10.3389/fnagi.2016.00184

Arenaza-Urquijo, E. M., Wirth, M., and Chételat, G. (2015). Cognitive reserve and lifestyle: moving towards preclinical Alzheimer's disease. Front. Aging Neurosci. 7:134. doi: 10.3389/fnagi.2015.00134

Baer, R. (2019). Assessment of mindfulness by self-report. Curr. Opin. Psychol. 28, 42-48. doi: 10.1016/j.copsyc.2018.10.015

Barnby, J. M., Bailey, N. W., Chambers, R., and Fitzgerald, P. B. (2015). How similar are the changes in neural activity resulting from mindfulness practice in contrast to spiritual practice? Conscious. Cogn. 36, 219-232. doi: 10.1016/j. concog.2015.07.002

Bennett, D. A., Buchman, A. S., Boyle, P. A., Barnes, L. L., Wilson, R. S., and Schneider, J. A. (2018). Religious orders study and rush memory and aging project. J. Alzheimers Disord. 64(Suppl. 1), S161-S189. doi: 10.3233/JAD179939

Binder, J. C., Bezzola, L., Haueter, A. I., Klein, C., Kühnis, J., Baetschmann, H., et al. (2017). Expertise-related functional brain network efficiency in healthy older adults. BMC Neurosci. 18:2. doi: 10.1186/s12868-016-0324-1

Borelli, W. V., Carmona, K. C., Studart-Neto, A., Nitrini, R., Caramelli, P., and da Costa, J. C. (2018). Operationalized definition of older adults with high cognitive performance. Dement. Neuropsychol. 12, 221-227. doi: 10.1590/198057642018dn12-030001

Bott, N. T., Bettcher, B. M., Yokoyama, J. S., Frazier, D. T., Wynn, M., Karydas, A., et al. (2017). Youthful processing speed in older adults: genetic, biological, and behavioral predictors of cognitive processing speed trajectories in aging. Front. Aging Neurosci. 9:55. doi: 10.3389/fnagi.2017.00055

Bowen, C. E., and Luy, M. (2018). Community social characteristics and health at older ages: evidence from 156 religious communities. J. Gerontol. Ser. B 73, 1429-1438. doi: 10.1093/geronb/gbw114

Boyle, P. A., Yang, J., Yu, L., Leurgans, S. E., Capuano, A. W., Schneider, J. A., et al. (2017). Varied effects of age-related neuropathologies on the trajectory of late life cognitive decline. Brain 140, 804-812. doi: 10.1093/brain/aww341

Caspersen, C. J., Powell, K. E., and Christenson, G. M. (1985). Physical activity, exercise, and physical fitness: definitions and distinctions for health-related research. Public Health Rep. 100, 126-131.

Christie, G. J., Hamilton, T., Manor, B. D., Farb, N. A., Farzan, F., Sixsmith, A., et al. (2017). Do lifestyle activities protect against cognitive decline in aging? A Review. Front. Aging Neurosci. 9:3. doi: 10.3389/fnagi.2017.00381

Clare, L., Wu, Y. T., Teale, J. C., MacLeod, C., Matthews, F., Brayne, C., et al. (2017). Potentially modifiable lifestyle factors, cognitive reserve, and cognitive function in later life: a cross-sectional study. PLoS Med. 14:e1002259. doi: 10. 1371/journal.pmed.1002259

Dayan, E., and Cohen, L. G. (2011). Neuroplasticity subserving motor skill learning. Neuron 72, 443-454. doi: 10.1016/j.neuron.2011.10.008

Dionigi, R., Baker, J., and Horton, S. (2011). Older athletes' perceived benefits of competition. Int. J. Sport Soc. 2, 17-22.

Dixon, R. A., and Lachman, M. E. (2019, forthcoming). "Risk and protective factors in cognitive aging: advances in assessment, prevention, and promotion of alternative pathways," in The Aging Brain: Functional Adaptation Across Adulthood. ed. G. Samanez-Larkin (Washington, DC: American Psychological Association).

Dupuy, O., Bosquet, L., Fraser, S. A., Labelle, V., and Bherer, L. (2018). Higher cardiovascular fitness level is associated to better cognitive dual-task performance in master athletes: mediation by cardiac autonomic control. Brain Cogn. 125, 127-134. doi: 10.1016/j.bandc.2018.06.003

Eriksen, B. A., and Eriksen, C. W. (1974). Effects of noise letters on the identification of a target letter in a non-search task. Percept. Psychophys. 16, 143-149. doi: 10.3758/bf03203267

Fien, S., Climstein, M., Quilter, C., Buckley, G., Henwood, T., Grigg, J., et al. (2017). Anthropometric, physical function and general health markers of Masters athletes: a cross-sectional study. PeerJ 5:e3768. doi: 10.7717/peerj. 3768
Gajewski, A. K., and Poznańska, A. (2008). Mortality of top athletes, actors and clergy in Poland: 1924-2000 follow-up study of the longer term effect of physical activity. Eur. J. Epidemiol. 23, 335-340. doi: 10.1007/s10654-008-9237-3

Geard, D., Reaburn, P., Rebar, A., and Dionigi, R. (2017). Masters athletes: exemplars of successful aging? J. Aging Phys. Activ. 25, 490-500. doi: 10.1123/ japa.2016-0050

Gefen, T., Shaw, E., Whitney, K., Martersteck, A., Stratton, J., Rademaker, A., et al. (2014). Longitudinal neuropsychological performance of cognitive SuperAgers. J. Am. Geriatr. Soc. 62, 1598-1600. doi: 10.1111/jgs.12967

Geiger, P. J., Boggero, I. A., Brake, C. A., Caldera, C. A., Combs, H. L., Peters, J. R., et al. (2016). Mindfulness-based interventions for older adults: a review of the effects on physical and emotional well-being. Mindfulness 7, 296-307. doi: 10.1007/s12671-015-0444-1

Gheysen, F., Poppe, L., DeSmet, A., Swinnen, S., Cardon, G., De Bourdeaudhuij, I., et al. (2018). Physical activity to improve cognition in older adults: can physical activity programs enriched with cognitive challenges enhance the effects? A systematic review and meta-analysis. Int. J. Behav. Nutr. Phys. Activ. 15:63. doi: 10.1186/s12966-018-0697-x

Giordano, J., and Engebretson, J. (2006). Neural and cognitive basis of spiritual experience: biopsychosocial and ethical implications for clinical medicine. Explore 2, 216-225. doi: 10.1016/j.explore.2006.02.002

Godde, B., and Voelcker-Rehage, C. (2017). Cognitive resources necessary for motor control in older adults are reduced by walking and coordination training. Front. Hum. Neurosci. 11:156. doi: 10.3389/fnhum.2017.00156

Gonçalves, J., Lucchetti, G., Menezes, P. R., and Vallada, H. (2017). Complementary religious and spiritual interventions in physical health and quality of life: a systematic review of randomized controlled clinical trials. PloS One 12:e0186539. doi: 10.1371/journal.pone.0186539

Grier, J. B. (1971). Nonparametric indexes for sensitivity and bias: computing formulas. Psychol. Bull. 75, 424-429. doi: 10.1037/h0031246

Harrison, T. M., Weintraub, S., Mesulam, M.-M., and Rogalski, E. (2012). Superior memory and higher cortical volumes in unusually successful cognitive aging. J. Int. Neuropsychol. Soc. 18, 1081-1085. doi: 10.1017/S1355617712000847

Huy, C., and Schneider, S. (2008). Instrument for the assessment of middleaged and older adults' physical activity: design, eliability and application of the German-PAQ-50+. Zeitschrift fuer Gerontologie \& Geriatrie 41, 208-216. doi: 10.1007/s00391-007-0474-y

Keadle, S. K., McKinnon, R., Graubard, B. I., and Troiano, R. P. (2016). Prevalence and trends in physical activity among older adults in the United States: a comparison across three national surveys. Prevent. Med. 89, 37-43. doi: 10. 1016/j.ypmed.2016.05.009

Kenny, R. A., Coen, R. F., Frewen, J., Donoghue, O. A., Cronin, H., and Savva, G. M. (2013). Normative values of cognitive and physical function in older adults: findings from the Irish longitudinal study on ageing. J. Am. Geriatr. Soc. 61(Suppl. 2), S279-S290. doi: 10.1111/jgs.12195

Koenig, H. G. (2012). Religion, spirituality, and health: a review and update. $A d v$. Mind-Body Med. 29, 19-26.

Latimer, C. S., Keene, C. D., Flanagan, M. E., Hemmy, L. S., Lim, K. O., White, L. R., et al. (2017). Resistance to Alzheimer disease neuropathologic changes and apparent cognitive resilience in the Nun and Honolulu-Asia aging studies. J. Neuropathol. Exp. Neurol. 76, 458-466. doi: 10.1093/jnen/nlx030

Lazarus, N. R., and Harridge, S. (2017). Declining performance of master athletes: silhouettes of the trajectory of healthy human ageing? J. Physiol. 595, 2941-2948. doi: 10.1113/JP272443

Leys, C., Ley, C., Klein, O., Bernard, P., and Licata, L. (2013). Detecting outliers: do not use standard deviation around the mean, use absolute deviation around the median. J. Exp. Soc. Psychol. 49, 764-766. doi: 10.1016/j.jesp.2013. 03.013

Lövdén, M., Bäckman, L., and Lindenberger, U. (2017). “The link of intellectual engagement to cognitive and brain aging," in Cognitive Neuroscience of Aging: Linking Cognitive and Cerebral Aging, eds R. Cabeza, L. Nyberg, and D. C. Park (Oxford: Oxford University Press), 461-484. doi: 10.1093/acprof:oso/ 9780199372935.003.0019

Malinowski, P., Moore, A. W., Mead, B. R., and Gruber, T. (2015). Mindful Aging: the effects of regular brief mindfulness practice on electrophysiological markers of cognitive and affective processing in older adults. Mindfulness 8, 78-94. doi: 10.1007/s12671-015-0482-8 
Marques, A., Sarmento, H., Martins, J., and Saboga Nunes, L. (2015). Prevalence of physical activity in European adults - compliance with the world health organization's physical activity guidelines. Prevent. Med. 81, 333-338. doi: 10.1016/j.ypmed.2015.09.018

McKendry, J., Breen, L., Shad, B. J., and Greig, C. A. (2018). Muscle morphology and performance in master athletes: a systematic review and meta-analyses. Ageing Res. Rev. 45, 62-82. doi: 10.1016/j.arr.2018.04.007

Miller, L., Bansal, R., Wickramaratne, P., Hao, X., Tenke, C. E., Weissman, M. M., et al. (2014). Neuroanatomical correlates of religiosity and spirituality: a study in adults at high and low familial risk for depression. JAMA Psychiatry 71, 128-135. doi: 10.1001/jamapsychiatry.2013.3067

Muehsam, D., Lutgendorf, S., Mills, P. J., Rickhi, B., Chevalier, G., Bat, N., et al. (2017). The embodied mind: a review on functional genomic and neurological correlates of mind-body therapies. Neurosci. Biobehav. Rev. 73, 165-181. doi: 10.1016/j.neubiorev.2016.12.027

Mulder, M., Ranchor, A. V., Sanderman, R., Bouma, J., and van den Heuvel, W. J. (1998). The stability of lifestyle behaviour. Int. J. Epidemiol. 27, 199-207. doi: 10.1093/ije/27.2.199

Nasreddine, Z. S., Phillips, N. A., Bedirian, V., Charbonneau, S., Whitehead, V., Collin, I., et al. (2005). The montreal cognitive assessment, MoCA: a brief screening tool for mild cognitive impairment. J. Am. Geriatr. Soc. 53, 695-699. doi: 10.1111/j.1532-5415.2005.53221.x

Negash, S., Wilson, R. S., Leurgans, S. E., Wolk, D. A., Schneider, J. A., Buchman, A. S., et al. (2013). Resilient brain aging: characterization of discordance between Alzheimer's disease pathology and cognition. Curr. Alzheimer Res. 10, 844-851. doi: 10.2174/15672050113109990157

Newberg, A. (2017). “The spiritual brain: science and religious experience," in The Physics of the Mind and Brain Disorders. Springer Series in Cognitive and Neural Systems. Vol. 11, eds I. Opris and M. Casanova (Cham: Springer), 649-670.

Nilson, J., and Lövdén, M. (2018). Naming is not explaining: future directions for the "cognitive reserve" and "brain maintenance" theories. Alzheimer's Res. Ther. 10:34. doi: 10.1186/s13195-018-0365-z

Norman, J. E., Rutkowsky, J., Bodine, S., and Rutledge, J. C. (2018). The potential mechanisms of exercise-induced cognitive protection: a literature review. Curr. Pharm. Design 24, 1827-1831. doi: 10.2174/13816128246661804061 05149

Park, D. C., and Reuter-Lorenz, P. (2009). The adaptive brain: aging and neurocognitive scaffolding. Annu. Rev. Psychol. 60, 173-196. doi: 10.1146/ annurev.psych.59.103006.093656

Petersen, K. E., Johnsen, N. F., Olsen, A., Albieri, V., Olsen, L. K., Dragsted, L. O., et al. (2015). The combined impact of adherence to five lifestyle factors on all-cause, cancer and cardiovascular mortality: a prospective cohort study among Danish men and women. Br. J. Nutr. 113, 849-858. doi: 10.1017/ S0007114515000070

Picavet, H. S. J., Wendel-Vos, G. C. W., Vreeken, H. L., Schuit, A. J., and Verschuren, W. M. M. (2011). How stable are physical activity habits among adults? the Doetinchem Cohort Study. Med. Sci. Sport Exerc. 43, 74-79. doi: 10.1249/MSS.0b013e3181e57a6a

Pignolo, R. J. (2018). Exceptional human longevity. Mayo Clin. Proc. 94, 110-124. doi: 10.1016/j.mayocp.2018.10.005

Raichlen, D. A., and Alexander, G. E. (2017). Adaptive capacity: an evolutionary neuroscience model linking exercise, cognition, and brain health. Trends Neurosci. 40, 408-421. doi: 10.1016/j.tins.2017.05.001

Ravaglia, R., Forti, P., Lucicesare, A., Pisacane, N., Rietti, E., and Patterson, C. (2008). Development of an easy prognostic score for frailty outcomes in the aged. Age Ageing 37, 161-166. doi: 10.1093/ageing/afm 195

Rea, I. M. (2017). Towards ageing well: use it or lose it: exercise, epigenetics and cognition. Biogerontology 18, 679-691. doi: 10.1007/s10522-017-9719-3

Reuter-Lorenz, P. A., and Park, D. C. (2014). How does it STAC up? Revisiting the scaffolding theory of aging and cognition. Neuropsychol. Rev. 24, 355-370. doi: 10.1007/s11065-014-9270-9

Rikli, R. E., and Jones, C. J. (2013). Senior Fitness Test Manual, 2nd Edn. Champaign, IL: Human Kinetics.

Rowe, J. W., and Kahn, R. L. (1997). Successful aging. Gerontologist 37, 433-441.

Satz, P. (1993). Brain reserve capacity on symptom onset after brain injury: a formulation and review of evidence for threshold theory. Neuropsychology 7 , 273-295. doi: 10.1037//0894-4105.7.3.273
Schienkiewitz, A., Mensink, G. B. M., Kuhnert, R., and Lange, C. (2017). Übergewicht und Adipositas bei Erwachsenen in Deutschland. J. Health Monit. 2, 21-28. doi: 10.17886/RKI-GBE-2017-025

Schott, N., El-Rajab, I., and Klotzbier, T. (2016). Cognitive-motor interference during fine and gross motor tasks in children with developmental coordination disorder. Res. Dev. Disabil. 57, 136-148. doi: 10.1016/j.ridd.2016.07.003

Snowdon, D. A. (2003). Healthy aging and dementia: findings from the nun study. Ann. Intern. Med. 139, 450-454. doi: 10.7326/0003-4819-139-5_Part_2200309021-00014

Stern, Y. (2002). What is cognitive reserve? Theory and research application of the reserve concept. J. Int. Neuropsychol. Soc. 8, 448-460. doi: 10.1017/ s1355617702813248

Sun, F. W., Stepanovic, M. R., Andreano, J., Barrett, L. F., Touroutoglou, A., and Dickerson, B. C. (2016). Youthful brains in older adults: preserved neuroanatomy in the default mode and salience networks contributes to youthful memory in superaging. J. Neurosci. 36, 9659-9668. doi: 10.1523/ JNEUROSCI.1492-16.2016

Taran, S., Taivassalo, T., and Sabiston, C. M. (2013). The neuroprotective effects of long-term exercise training in older adults: a look at world-ranking elite Masters athletes. J. Exerc. Move. Sport 45:192.

Thomas, B. P., Yezhuvath, U. S., Tseng, B. Y., Liu, P., Levine, B. D., Zhang, R., et al. (2013). Life-long aerobic exercise preserved baseline cerebral blood flow but reduced vascular reactivity to CO2. J. Magn. Resonan. Imaging 38, 1177-1183. doi: 10.1002/jmri.24090

Tseng, B. Y., Uh, J., Rossetti, H. C., Cullum, C. M., Diaz-Arrastia, R. F., Levine, B., et al. (2013). Masters athletes exhibit larger regional brain volume and better cognitive performance than sedentary older adults. J. Magn. Resonan. Imaging 38, 1169-1176. doi: 10.1002/jmri.24085

Tyndall, A. V., Clark, C. M., Anderson, T. J., Hogan, D. B., Hill, M. D., and Poulin, M. J. (2018). Protective effects of exercise on cognition and brain health in older adults. Exerc. Sport Sci. Rev. 46, 215-223. doi: 10.1249/JES.0000000000000161

van Leeuwen, S., Singer, W., and Melloni, L. (2012). Meditation increases the depth of information processing and improves the allocation of attention in space. Front. Hum. Neurosci. 6:133. doi: 10.3389/fnhum.2012.00133

Weng, P.-H., Chen, J.-H., Chiou, J.-M., Tu, Y.-K., Chen, T.-F., Chiu, M.J., et al. (2018). The effect of lifestyle on late-life cognitive change under different socioeconomic status. PLoS One 13:e0197676. doi: 10.1371/journal. pone.0197676

Wiedemann, A., Marcher, A., Wegner-Siegmundt, C., Di Giulio, P., and Luy, M. (2014). Der Gesundheits-Survey der Klosterstudie. Daten- und Methodenbericht zu Welle 1 [The health survey of the Cloister Study. Data and methods report for wave 1], Vol. 37. Vienna: Austrian Academy of Sciences.

Wilson, R. S., and Bennett, D. A. (2017). How does psychosocial behavior contribute to cognitive health in old age? Brain Sci. 7:56. doi: 10.3390/ brainsci7060056

Wilson, R. S., Segawa, E., Buchman, A. S., Boyle, P. A., Hizel, L. P., and Bennett, D. A. (2012). Terminal decline in motor function. Psychol. Aging 27, 998-1007. doi: $10.1037 / \mathrm{a} 0028182$

Wouters, H., Aalbers, T., Maessen, M. F. H., Verbeek, A. L. M., Rikkert, M. G. M. O., Kessels, R. P. C., et al. (2017). Physical activity and cognitive function of long-distance walkers: studying four days marches participants. Rejuvenation Res. 20, 367-374. doi: 10.1089/rej.2016.1876

Wu, C., Pontifex, M. B., Raine, L. B., Chaddock, L., Voss, M. W., Kramer, A. F., et al. (2011). Aerobic fitness and response variability in preadolescent children. Neuropsychology. 25, 333-341. doi: 10.1037/a0022167

Yesavage, J. A., Brink, T. L., Rose, T. L., Lum, O., Huang, V., Adey, M., et al. (1983). Development and validation of a geriatric depression screening scale: a preliminary report. J. Psychiatr. Res. 17, 37-49. doi: 10.1016/0022-3956(82) 90033-4

Young, J., Angevaren, M., Rusted, J., and Tabet, N. (2015). Aerobic exercise to improve cognitive function in older people without known cognitive impairment. Cochrane Database Syst. Rev. CD005381. doi: 10.1002/14651858. CD005381.pub4

Yu, Y., Collinson, S. L., Liew, T. M., Ng, T.-P., Mahendran, R., Kua, E.-H., et al. (2019). Super-cognition in aging: cognitive profiles and associated lifestyle factors. Appl. Neuropsychol. Adult 1-7. doi: 10.1080/23279095.2019.15 70928 
Yun, R. J., Krystal, J. H., and Mathalon, D. H. (2010). Working memory overload: fronto-limbic interactions and effects on subsequent working memory function. Brain Imaging Behav. 4, 96-108. doi: 10.1007/s11682-010-9089-9

Zahodne, L. B., Nowinski, C. J., Gershon, R. C., and Manly, J. J. (2014). Which psychosocial factors best predict cognitive performance in older adults? J. Int. Neuropsychol. Soc. 20, 487-495. doi: 10.1017/S1355617714000186

Zhao, E., Tranovich, M. J., DeAngelo, R., Kontos, A. P., and Wright, V. J. (2016). Chronic exercise preserves brain function in master athletes when compared to sedentary counterparts. Phys. Sports Med. 44, 8-13. doi: 10.1080/00913847. 2016.1103641
Conflict of Interest Statement: The authors declare that the research was conducted in the absence of any commercial or financial relationships that could be construed as a potential conflict of interest.

Copyright (c) 2019 Schott and Krull. This is an open-access article distributed under the terms of the Creative Commons Attribution License (CC BY). The use, distribution or reproduction in other forums is permitted, provided the original author(s) and the copyright owner(s) are credited and that the original publication in this journal is cited, in accordance with accepted academic practice. No use, distribution or reproduction is permitted which does not comply with these terms. 